\title{
Quality Optimization of Data Rate Video Streaming Over Bluetooth Network
}

\author{
Andi Hasad ${ }^{1, *}$ Muhammad Amin Bakri ${ }^{1}$ Abdul Hafid Paronda ${ }^{1}$ Sri Marini ${ }^{1}$ \\ ${ }^{1}$ Electronic, Universitas Islam 45, 17113, Indonesia \\ *Corresponding author.Email: andihasad@gmail.com
}

\begin{abstract}
Video streaming over Bluetooth network as a medium of learning at higher education in the world began in 2004, in Indonesia, it began in 2012. Unfortunately, the development of video streaming using Bluetooth network has not been used optimally in the field of higher education, especially at client-server distances reaching $50 \mathrm{~m}$. This is caused by the video quality received from the client is not good and not according to Cisco standards. This paper proposes a solution by providing a good video quality based on optimizing the performance of video streaming data rate transmission over Bluetooth Piconet Pervasive network, with Android OS on the client-side. This research is quantitative research with an experimental method. All average values of throughput, delay, jitter, and packet loss parameters are eligible for Cisco standard video streaming for $50 \mathrm{~m}$ measurements, where packet loss is below $5 \%$, the value of packet loss parameters obtained has the highest value of $4.97 \%$, and the lowest of $4.88 \%$ with an average value of $4.92 \%$. This research contributed to providing a good quality of video streaming over Bluetooth networks. The results showed that the greater the interference of Wi-Fi on the Bluetooth piconet pervasive network, the quality of video received by the client (mobile phone) decreased, marked by the increasing value of the average packet loss obtained during the video streaming.
\end{abstract}

Keywords: Bluetooth, Quality Optimization, Data Rate Video Streaming, Piconet Pervasive, Client-Server.

\section{INTRODUCTION}

Research on Bluetooth networks in the field of information and communication technology or the field of electrical engineering as a supporting learning infrastructure has been developed. This development research addressed the performances of Bluetooth voice communication system under interference and simulates Bluetooth voice communication system using Matlab Simulink [1], streaming video using a Bluetooth network on a computer with a Linux operating system, resulting in the time needed for streaming video transmission, the greater the size of the data packet sent [2]. This study also resulted in the difference in the Bluetooth version on the cellular phone side, influencing the number of data packets that could be received by the cellular telephone. Video Streaming over 802,11b in the presence of fading due to human traffic and Bluetooth interference conducted in 2009 [3], and an adaptive approach for video Streaming and evaluation over Bluetooth network in 2012 [4]. A system was developed to make it easier for students to understand the working principle of video transmission using Bluetooth networks, by testing various video streaming transmissions using Bluetooth networks on cellular phones to stream video clips and real-time videos from cellular phones to computers and from computers to cellular phones, using the Java platform [5]. As a result, the quality of the video sent decreases with increasing distance and the presence of Wi-Fi interference. The research in 2017, resulted, recommending that for peer-to-peer and client-server communications carried out on 1 channel, the protocol that can be used is RFCOMM, while for many clients, using point to multipoint topology, the protocol used is L2CAP [6].

Some ways to deal with the weakness of video streaming using Bluetooth networks include video compression, Quality of Service (QoS) control, and intermediate protocols. Video compression is used to eliminate excess video data information, thereby increasing efficiency in Bluetooth network transmission. QoS which includes congestion control and error control is used to handle packet loss, reduce delay and improve video quality, while intermediate protocols are used to split video data into packets before being sent [7]. The research that collects data through investigations to their students about their perception of Bluetooth security, 
resulting in communication via Bluetooth can be safe but still careful in its use. This study suggests that learning about Bluetooth communication technology be included in the college curriculum [8]. The Bluetooth system can be integrated into Android, which is the main cellular phone operating system as a mobile communication tool today. This study also developed a chat application using 2-way communication on a Bluetooth network, without other infrastructure. This research is used to develop learning processes based on Bluetooth networks [9].

Previous research on a Bluetooth Piconet Pervasive system, it produces the best performance from the system in transmitting data rates via Bluetooth networks. But the research still runs on the Symbian platform on the client side [10]. Therefore, in this research Piconet Pervasive system was developed, which runs on the Android opensource operating system on the client side; which is a cellular phone operating system that is widely used today. The system produces the best performance on the transmission of real-time video streaming data rates through the Bluetooth Piconet Pervasive network, according to Cisco streaming video standards. The design is based on Bluetooth Low Energy (BLE) communication with peripheral devices [11].

The purpose of this study was to develop a Piconet Pervasive system that uses a client-server connection point to multi-point topology using the Android operating systems on the client-side (phones), which meet the video streaming QoS standards, both when transmitting video streaming data rates and on the system Piconet Pervasive, in environments that have $\mathrm{Wi}-\mathrm{Fi}$ interference or that do not have $\mathrm{Wi}-\mathrm{Fi}$ interference.

\section{METHOD}

The research method includes several stages of research, including Piconet Pervasive Bluetooth network system analysis, system design, audio-video compression, hint track, optimization, system testing, performance measurement, and analysis of measurement results, system optimization, and implementation.

\subsection{Piconet Pervasive Bluetooth System Analysis}

The Pervasive Piconet system is a piconet system that connects two different devices so that the existence of these different devices is no longer felt. At this stage, identification of the needs of the Bluetooth Piconet Pervasive network system. Needs identification is based on literature studies and literature on hardware and software needed during the research. Literature studies also include multimedia on cellular phones, audio-video processing, Bluetooth connections from cellular phones to computers, and from computers to cellular phones.

\subsection{System Design}

At this stage, prototype design and construction are carried out for Bluetooth connections from the computer (server) to the cellular telephone (client). The design and configuration carried out on the server-side consist of software and hardware.

\subsection{Audio Video Compression}

The compression process is used to reduce the video data rate. Data must be compressed before sending it via a Bluetooth network. The compressed data consists of two parts, namely audio and video, including frame size, frame rate, codec, audio rate, sample rate, and channels. The video that has been compressed is then sent via a Bluetooth network with limited bandwidth.

\subsection{Hint Track}

Before the video is sent, the hint track process is first done so that the video can be run/played on the client video player. The hint track process is needed to give information to the video so that it is ready to be sent and can be recognized by the client. The bandwidth obtained from this process is the limit of videos that can be sent.

\subsection{Optimization}

This stage has done when the system can work but has not yet produced the expected value based on Cisco's standard video streaming QoS [12]. Optimization on the server has done by tuning up to maximize server performance, while on the client-side optimization was done by minimizing running background, which consumes memory on cell phones.

\subsection{System Testing}

System testing aims to determine the ability of the Bluetooth network as a video streaming media and to find out the quality of the video received on the client-side. The protocol used is RFCOMM with an intermediate protocol in the form of Internet Protocol (IP). The video data rate tested is $0.8 \mathrm{Mbps}$. 1.6 Mbps and 2.0 Mbps, while the distance to be tested is 50 meters.

\subsection{Performance Measurement}

The parameters used in measuring the performance of this Bluetooth network are throughput, delay, jitter, and packet loss. Measurement of this parameter uses capture network traffic. 


\section{RESULT AND DISCUSSION}

\subsection{Result}

The measurement starts with sending a video that is $50 \mathrm{~m}$ away from the server, in an environment that does not have Wi-Fi $(-100 \mathrm{dBm})$, and an environment that has Wi-Fi signal strength of $-78 \mathrm{dBm}$ and $-58 \mathrm{dBm}$. After each video is measured, then comparison and analysis of the measurement parameters between the videos are carried out. The results of measuring video streaming at $0.8 \mathrm{Mbps}$ data rate and $50 \mathrm{~m}$ client-server distance as shown in Table 1.
Based on Table 1, it can be seen that in an environment that does not have Wi-Fi $(-100 \mathrm{dBm})$, the highest value for throughput parameters is 3.54 packets / second and the lowest value is 3.49 packets / second with an average of 3.51 packets/second. The delay parameter has the highest value of 0.69 milliseconds and the lowest value is 0.67 milliseconds with an average value of 0.68 milliseconds. For the jitter parameter, the highest value is 0.02 milliseconds and the lowest is 0.01 milliseconds with an average value of 0.02 milliseconds. The packet loss parameter has the highest value of $4.38 \%$ and the lowest value is $4.34 \%$ with an average value of $4.36 \%$.

Table 1. The measurement results carried out on the client-server distance are $50 \mathrm{~m}$, data rate $0.8 \mathrm{Mbps}$

\begin{tabular}{|c|c|c|c|c|c|c|c|c|c|c|c|c|c|c|c|}
\hline \multirow{2}{*}{ Parameters } & \multicolumn{5}{|c|}{ Wi-fi : -100 dBm } & \multicolumn{4}{|c|}{ Wi-fi : - $78 \mathrm{dBm}$} & \multicolumn{6}{|c|}{ Wi-fi : $-58 \mathrm{dBm}$} \\
\hline & M-1 & M-2 & M-3 & M-4 & Average & M-1 & M-2 & M-3 & M-4 & Average & M-1 & M-2 & M-3 & M-4 & Average \\
\hline $\begin{array}{l}\text { Throughput } \\
\text { (packet/second) }\end{array}$ & 3.54 & 3.49 & 3.49 & 3.51 & 3.51 & 3.42 & 3.41 & 3.39 & 3.51 & 3.43 & 3.32 & 3.33 & 3.28 & 3.33 & 3.32 \\
\hline $\begin{array}{l}\text { Delay } \\
\text { (millisecond) }\end{array}$ & 0.69 & 0.67 & 0.69 & 0.68 & 0.68 & 0.89 & 0.87 & 0.91 & 0.88 & 0.89 & 0.96 & 0.98 & 0.95 & 0.99 & 0.97 \\
\hline $\begin{array}{l}\text { Jitter } \\
\text { (millisecond) }\end{array}$ & 0.02 & 0.02 & 0.01 & 0.01 & 0.02 & 0.02 & 0.04 & 0.03 & 0.03 & 0.03 & 0.02 & 0.03 & 0.04 & 0.04 & 0.03 \\
\hline $\begin{array}{l}\text { Packet Loss } \\
(\%)\end{array}$ & 4.35 & 4.34 & 4.38 & 4.38 & 4.36 & 4.69 & 4.66 & 4.65 & 4.68 & 4.67 & 4.97 & 4.93 & 4.88 & 4.91 & 4.92 \\
\hline
\end{tabular}

In the delay parameter, the value obtained is in milliseconds, with the highest value of 0.69 milliseconds, this is following Cisco's QoS standard which allows a maximum of 5 seconds to stream video streaming. While the jitter parameters in video streaming do not have standard standards because streaming video is not jitter sensitive based on criteria issued by Cisco. The Jitter parameter is closely related to the delay parameter, and the jitter parameter can be used to determine the stability of the data packet transmission.

In packet loss parameters, the highest value of packet loss is $4.38 \%$, where the average value is $4.36 \%$. This value is still included in the standard for streaming video based on Cisco QoS, where the standard value that is still allowed is $<5 \%$.

In environments that have Wi-Fi $(-78 \mathrm{dBm}$ and -58 $\mathrm{dBm})$, the highest value for throughput parameters is 3.51 packets / second and the lowest value is 3.28 packets / second with an average of 3.43 packets / second in the environment with a $\mathrm{Wi}-\mathrm{Fi}$ signal of $-78 \mathrm{dBm}$ and 3.32 packets / second in an environment with a Wi-Fi signal of $-58 \mathrm{dBm}$. This shows that the stronger the $\mathrm{Wi}-\mathrm{Fi}$ interference, the lower the throughput value.
The delay parameter has the highest value of 0.99 milliseconds and the lowest value is 0.87 milliseconds with an average value of 0.89 milliseconds in an environment with a Wi-Fi signal of $-78 \mathrm{dBm}$ and 0.97 milliseconds in an environment with a $\mathrm{Wi}-\mathrm{Fi}$ signal of $58 \mathrm{dBm}$. These results indicate that the stronger the Wi$\mathrm{Fi}$ interference, the greater the delay. For jitter parameters, the highest value is 0.04 milliseconds and the lowest is 0.02 milliseconds with an average value of 0.32 milliseconds in an environment with a Wi-Fi signal of $78 \mathrm{dBm}$ and 0.03 milliseconds in an environment with a $\mathrm{Wi}-\mathrm{Fi}$ signal of $-58 \mathrm{dBm}$. The difference in the numbers obtained by the relatively small difference in value indicates the stability of the network used.

In an environment with a $\mathrm{Wi}-\mathrm{Fi}$ signal of $-78 \mathrm{dBm}$, packet loss parameter values obtained have the highest value of $4.69 \%$ and the lowest is $4.65 \%$ with an average value of $4.67 \%$, while in environments with $\mathrm{Wi}-\mathrm{Fi}$ signal $-58 \mathrm{dBm}$, the value packet loss parameters obtained have the highest value of $4.97 \%$ and the lowest is $4.88 \%$ with an average value of $4.92 \%$. This shows that the stronger the Wi-Fi interference on the network, the greater the value of packet loss. 

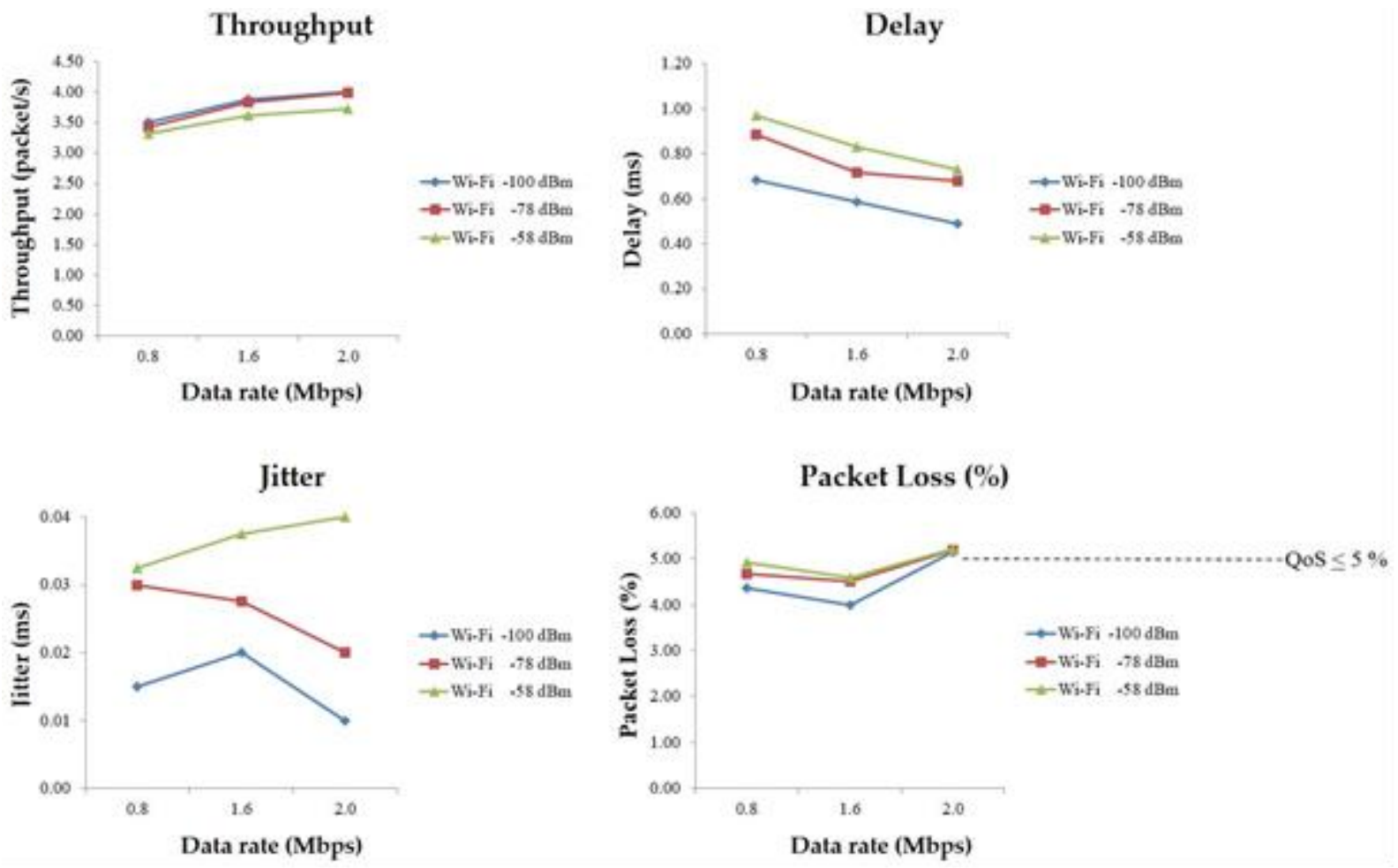

Figure 1 Comparison of measurement results at $0.8 \mathrm{Mbps}, 1.6 \mathrm{Mbps}$, and 2.0 Mbps data rates

All data of delay and packet loss, which was obtained in this research have met Cisco streaming video standards; with a standard maximum packet loss is $5 \%$ for a data rate $0.8 \mathrm{Mbps}$. The value of packet loss parameters obtained has the highest value of $4.97 \%$ and the lowest of $4.88 \%$ with an average value of $4.92 \%$, in an environment that has $\mathrm{Wi}-\mathrm{Fi}$, the client-server distance $50 \mathrm{~m}$, that is by doing optimization to get the best performance on the Piconet Pervasive system. Figure 1 shows that on the other side of the measurement, for data rates of 2.0 MBps, we found some errors, especially for environments that have wi-fi interference of $-78 \mathrm{dBm}$ and $-58 \mathrm{dBm}$, which means getting packet loss $>5 \%$.

\subsection{Discussion}

The results showed that the greater the interference of Wi-Fi on the Bluetooth Piconet Pervasive network, the quality of video received by the client (phones) decreased, marked by the increasing value of the average packet loss obtained during the video streaming. The results indicate that the stronger the $\mathrm{Wi}-\mathrm{Fi}$ interference, the greater the delay. The Jitter parameter is closely related to the delay parameter, the jitter parameter can be used to determine the stability of the data packet transmission, the closer the value of 0 is the more stable data transmission.

\section{CONCLUSION}

In this paper, we present the Piconent Pervasive System as a video streaming quality solution running on smartphones for almost seven years already. Our proposed Piconet Pervasive System uses a client-server architecture, communicating with the other phones via Bluetooth. In this case, we use at a certain location several phones, receives media content from the computer server. We wrote down also some considerations regarding fault tolerance, in an environment that has wi-fi interference, which does not meet the Cisco standard for video streaming Quality of Service.

\section{ACKNOWLEDGMENTS}

We would like to thank the Directorate General of Higher Education (DIKTI), Ministry of Education and Culture. We would also like to thank the anonymous reviewers of the Institutional National Strategy Research scheme in 2017.

\section{REFERENCES}

[1] W. Zhengzhong, Z. Xianxin, Y. Wenge and L. Zilin, "System-Level Simulation Modeling of Bluetooth Voice and It's Interference," ICSP'04 Proceedings, pg. 29-32, 2004.

[2] D. Catania and S. Zammit, "Video Streaming over Bluetooth," Dissertation, University of Malta, Malta, 2008.

[3] K. Eudon and B. R. Petersen, "Video Streaming over $802,11 \mathrm{~b}$ in the Presence of Fading due to Human Traffic and Bluetooth Interference", 
Seventh Annual Communication Networks and Services Conference, vol. 10, pg. 33-40, 2009.

[4] R. Bin Sattar, N. Ahmed, and M. Rahman. "An Adaptive Approach for Video Streaming and Evaluation over Bluetooth Network", 8th International Conference on Wireless Communications, Networking and Mobile Computing vol. 8, pg. 1-4, 2012.

[5] S. Gupta, S. Singh, and R. Jain, "Analysis and Optimization of Various Transmission Issues in Video Streaming over Bluetooth," International Journal of Computer Application vol. 11, pg. 44-48, 2010.

[6] C. Jung, K. Kim, J. Seo, N. Silva, and K. Han, "Topology Configuration and Multihop Routing Protocol for Bluetooth Low Energy Networks", IEEE Journals and Magazines (5): pg. 9587-9598, 2017.

[7] S. Banerjee, D. Mondal, D. Sumit, and R. Bikash Guin, "Real-Time Video Streaming Over Bluetooth Network Between Two Mobile Nodes," International Journal of Computer Science Issues, vol. 7, pg. 37-39, 2010.
[8] T. Margaret and S. Kathrine, "An Investigation of Students' Perception of Bluetooth Security," IEEE 2011 International Conference on Information Science and Applications, Vol. 22, No.2, pg. 269289, 2012.

[9] M. Mahajan, G. Verma, G. Erale, S. Bonde and D. Arya, "Design of Chatting Application Based on Android Bluetooth", IJCSMC, Vol. 3, Issue. 3, pg.712 - 717, India, 2014.

[10] D. Arnaldy, S. Wahjuni, and S. Guritman, "The Design of Piconet Pervasive System Architecture for Video Streaming Applications," Information and Communication Technology for the Muslim World (ICT4M), pg. E9-E12, 2010.

[11] M. Porjazoski, P. Latkoski, and B. Popovski, "Bluetooth Low Energy-based Smart Home Android Solution,” IEEE EUROCON 2019 - 18th International Conference on Smart Technologies, pg. 1-5, 2019.

[12] T. Szigeti and C. Hattingh, "End-to-End QoS Network Design: Quality of Service in LANs, WANs, and VPNs", Cisco Press, Indianapolis, 2004. 